SCIENTIFIC PUBLICATIONS OF THE STATE UNIVERSITY OF NOVI PAZAR

SER. A: Appl. MATH. INFORM. AND MECH. vol. 12, 2 2020, 57-66.

\title{
Generalised Regularity and Compactness of Matrix Operators
}

\author{
E. Malkowsky
}

\begin{abstract}
A well-known result by Cohen and Dunford ([2], 1937) characterises the class of all regular compact linear operators. It follows that a regular matrix transformation cannot be compact. This means that if $c$ denotes the set of all complex sequences of complex numbers, then an infinite matrix that maps $c$ into $c$ and preserves the limits cannot be compact. We obtained this result in a different way applying the theory of $B K$ spaces from functional analysis and summability, and using the Hausdorff measure of noncompactness. Furthermore, we present the extension of this result to matrix transformations between the spaces $c$ and the spaces of strongly summable sequences by the Cesàro method of order 1 , and of strongly convergent sequences. We present new unified proofs for our main results.
\end{abstract}

Keywords: Sequence spaces, bounded linear operators, Hausdorff measure of noncompactness, regular and compact operators

\section{Introduction and Notations}

Measures of noncompactness are very useful tools in functional analysis, for instance in metric fixed point theory and the theory of operator equations in Banach spaces. They can also be used in the characterisations of classes of compact bounded operators between $B K$ spaces by establishing identities or estimates for their Hausdorff measures of noncompactness. This approach was initiated on a large scale in [8], and later also presented in detail in $[9,10]$.

We consider the spaces of convergent sequences, of sequences that are strongly $C_{1}-$ summable with index $p \geq 1$, and of strongly convergent sequences denoted by $c, w^{p}$ and $[c]$, respectively, and apply the theory of $B K$ spaces to characterise the classes of matrix operators from $c, w^{p}$ and $[c]$ into $c$, and to determine their operator norms. Furthermore, we obtain the estimates for the Hausdorff measure of noncompactness of these operators, which yield the characterisations of the subclasses of compact matrix operators. Our main results are obtained by new unified results. They imply that matrix operators between those spaces that preserve the associated limits cannot be compact. They include the special case obtained by Cohen and Dunford [2].

Manuscript received May 15, 2020 ; accepted August 22, 2020

E. Malkowsky is with the University Union Nicola Tesla, Njegoševa 1a, 21205 Sremski Karlovci, Serbia. 
Now we list some of the used standard notations and results.

The set of all complex sequences $x=\left(x_{k}\right)_{k=1}^{\infty}$ is denoted by $\omega$; we write $\ell_{\infty}, c, c_{0}$ and $\phi$ for the subsets of $\omega$ of all bounded, convergent, null and finite sequences, and $\ell_{1}$ for the set of all absolutely convergent series.

Let $e=\left(e_{k}\right)_{k=1}^{\infty}$ and $e^{(n)}=\left(e_{k}^{(n)}\right)_{k=1}^{\infty}$ for $n \in \mathbb{N}$ denote the sequences with $e_{k}=1$ for all $k$, and $e_{n}^{(n)}=1$ and $e_{k}^{(n)}=0$ for $k \neq n$.

A $B K$ space $X$ is a Banach sequence space with the property that all the coordinates $P_{n}: X \rightarrow \mathbb{C}$ with $P_{n}(x)=x_{n}\left(x=\left(x_{k}\right)_{k=1}^{\infty} \in X\right)$ are continuous; a $B K$ space $X$ is said to have $A K$ if $x=\lim _{m \rightarrow \infty} x^{[m]}$ for all $x=\left(x_{k}\right)_{k=1}^{\infty} \in X$, where $x^{[m]}=\sum_{k=1}^{m} x_{k} e^{(k)}$ denotes the $m$-section of the sequence $x=\left(x_{k}\right)_{k=1}^{\infty} \in X$.

Let $X, Y \subset \omega$ and $A=\left(a_{n k}\right)_{n, k=1}^{\infty}$ be an infinite matrix of complex entries. The $\beta$-dual of $X$ is the set $X^{\beta}=\left\{a=\left(a_{k}\right)_{k=1}^{\infty} \in \omega: \sum_{k=1}^{\infty} a_{k} x_{k}\right.$ converges for all $\left.x=\left(x_{k}\right)_{k=1}^{\infty} \in X\right\}$. We write $A_{n}=\left(a_{n k}\right)_{k=1}^{\infty}(n \in \mathbb{N})$ and $A^{k}=\left(a_{n k}\right)_{n=1}^{\infty}(k \in \mathbb{N})$ for the sequences in the $n^{\text {th }}$ row and the $k^{\text {th }}$ column of $A, A_{n} x=\sum_{k=1}^{\infty} a_{n k} x_{k}$ for $n \in \mathbb{N}$ and $A x=\left(A_{n} x\right)_{n=1}^{\infty}$ for the $A$-transform of the sequence $x$ (provided all the series converge); $X_{A}=\{x \in \omega: A x \in X$ for all $x \in X\}$ denotes the matrix domain of $A$ in $X$, and $(X, Y)$ is the class of all matrix transformations from $X$ into $Y$, that is, $A \in(X, Y)$ if and only if $X \subset Y_{A}$, or equivalently, $A \in(X, Y)$ if and only if $A_{n} \in X^{\beta}$ for all $n \in \mathbb{N}$ and $A x \in Y$ for all $x \in X$.

Let $X$ and $Y$ be Banach spaces. Then we write, as usual, $\mathscr{B}(X, Y)$ for the Banach space of all bounded linear operators $L: X \rightarrow Y$ with the operator norm $\|L\|=\sup \{\|L(x)\|:$ $\|x\|=1\}$; if $Y=\mathbb{C}$, then $X^{*}=\mathscr{B}(X, \mathbb{C})$ denotes the continuous dual of $\mathrm{X}$ with the norm $\|f\|=\sup \{|f(x)|:\|x\|=1\}$. The relation between the classes $\mathscr{B}(X, Y)$ and $(X, Y)$ for $B K$ spaces $X$ and $Y$ well-known and summarized in the following result.

Theorem 1.1 Let $X$ and $Y$ be $B K$ spaces.

(a) ([12, Theorem 4.2.8]) Then $(X, Y) \subset \mathscr{B}(X, Y)$, that is, every $A \in(X, Y)$ defines an operator $L_{A} \in \mathscr{B}(X, Y)$, where

$$
L_{A}(x)=\text { Ax for all } x \in X .
$$

(b) ([3, Theorem 1.9]) If $X$ has $A K$ then $\mathscr{B}(X, Y) \subset(X, Y)$, that is, for each $L \in \mathscr{B}(X, Y)$, there exists a matrix $A \in(X, Y)$ such that

$$
A x=L(x) \text { for all } x \in X
$$

in this case we say that the matrix A represents the operator $L$.

\section{The spaces $w^{p}$ and $[c]$ and their $\beta$ - and continuous duals}

The sets $w^{p}$ of strongly $C_{1}$ summable sequences, with index $p \geq 1$ and the sets $[c]$ of strongly convergent sequences were studied by Maddox [5], and Kuttner and Thorpe [4], respectively.

Throughout, let $p \geq 1$. 
If $y=\left(y_{k}\right)_{k=1}^{\infty} \in \omega$, then we write $\Delta y_{k}=y_{k}-y_{k-1}$ with the convention $y_{0}=0$.

We write

$$
w^{p}=\left\{x \in \omega: \lim _{n \rightarrow \infty} \frac{1}{n} \sum_{k=1}^{n}\left|x_{k}-\xi_{w^{p}}\right|^{p}=0 \text { for some } \xi_{w^{p}} \in \mathbb{C}\right\}
$$

and

$$
[c]=\left\{x \in \omega: \lim _{n \rightarrow \infty} \frac{1}{n} \sum_{k=1}^{n}\left|\Delta\left(k x_{k}\right)-\xi_{[c]}\right|=0 \text { for some } \xi_{[c]} \in \mathbb{C}\right\} .
$$

The unique complex numbers $\xi_{w^{p}}$ and $\xi_{[c]}$ are referred to as the $w^{p}-$ and $[c]$-limits of the sequence $x$. We also use the notation $\xi_{c}$ for the usual limit of the sequence $x \in c$.

If $X \in\left\{c, w^{p},[c]\right\}$, then the norms $\|\cdot\|_{X}$ are defined by

$$
\|x\|_{X}= \begin{cases}\|x\|_{\infty}=\sup _{k}\left|x_{k}\right| & \text { for } X=c \\ \sup _{v}\left(\frac{1}{2^{v}} \sum_{k=2^{v}}^{2^{v+1}-1}\left|x_{k}\right|^{p}\right)^{1 / p} & \text { for } x=w^{p} \\ \sup _{v} \frac{1}{2^{v}} \sum_{k=2^{v}}^{2^{v+1}-1}\left|\Delta\left(k x_{k}\right)\right| & \text { for } x=[c] .\end{cases}
$$

If $X \in\left\{c, w^{p},[c]\right\}$, then we write $X_{0}$ for the set of all $x \in X$ with $\xi_{X}=0$.

The following result is well known, where the case $X=c$ is standard. The cases $X=w^{p}$ and $X=[c]$ are [8, Proposition 3.44] and [6, Theorem 2.2].

Proposition 2.1 Let $X \in\left\{c, w^{p},[c]\right\}$. Then we have:

(a) $X$ and $X_{0}$ are $B K$ spaces with the norm $\|\cdot\|_{X}$, and $X_{0}$ is a closed subspace of $X$;

(b) $X_{0}$ has $A K$;

(c) every sequence $x=\left(x_{k}\right)_{k=1}^{\infty} \in X$ has a unique representation

$$
x=\xi_{X} e+\sum_{k=1}^{\infty}\left(x_{k}-\xi_{X}\right) e^{(k)}
$$

We introduce the following notations for the $\beta$-duals of our spaces. We write $\max _{v}$ and $\sum_{v}$ for the maximum and sum taken over all indices $k$ from $2^{v}$ to $2^{v+1}-1(v=0,1, \ldots)$, and put $\mathscr{W}_{p}=\left\{a \in \omega:\|a\|_{\mathscr{M}_{p}}<\infty\right\}$ and $\mathscr{C}=\left\{a \in \omega:\|a\|_{\mathscr{C}}<\infty\right\}$, where

$$
\begin{aligned}
\|a\|_{\mathscr{W}_{p}} & = \begin{cases}\sum_{v=0}^{\infty} 2^{v} \max _{v}\left|a_{k}\right| & (p=1) \\
\sum_{v=0}^{\infty} 2^{v / p}\left(\sum_{v}\left|a_{k}\right|^{q}\right)^{1 / q} & (1<p<\infty ; q=p /(p-1)),\end{cases} \\
\|a\|_{\mathscr{C}} & =\sum_{v=0}^{\infty} 2^{v} \max _{v}\left|\sum_{j=k}^{\infty} \frac{a_{j}}{j}\right| ;
\end{aligned}
$$


we also use the notations

$$
\mathscr{X}=\left\{\begin{array}{ll}
\ell_{1} & (X=c) \\
\mathscr{W}_{p} & \left(X=w^{p}\right) \\
\mathscr{C} & (X=[c])
\end{array} \quad \text { and }\|a\|_{1}=\sum_{k=1}^{\infty}\left|a_{k}\right| \text { for } a \in \ell_{1} .\right.
$$

Let $a \in \omega$ and $X$ be a $B K$ space. Then we write

$$
\|a\|_{X}^{*}=\sup _{\|x\|=1}\left|\sum_{k=1}^{\infty} a_{k} x_{k}\right|
$$

provided the expression on the right exists and is finite, which is the case by [12, Theorem 7.2.9], whenever $a \in X^{\beta}$.

The following results are known, where the case $X=c$ is standard. The cases $X=w^{p}$ and $X=[c]$ are [8, Proposition 3.47] and [7, Theorem 2].

Proposition 2.2 Let $X \in\left\{c, w^{p},[c]\right\}$. Then we have:

(a) $X^{\beta}=X_{0}^{\beta}=\mathscr{X}, X_{0}^{*}$ and $\mathscr{X}$ are norm isomorphic, and $\|a\|_{w^{p}}^{*}=\|a\|_{\mathscr{X}}$ for all $x \in X$;

(b) $f \in X^{*}$ if and only if there exist $b \in \mathbb{C}$ and a sequence $a=\left(a_{k}\right)_{k=1}^{\infty} \in \mathscr{X}$ such that

$$
f(x)=\xi_{X} b+\sum_{k=1}^{\infty} a_{k} x_{k} \text { for all } x \in X
$$

where

$$
a=\left(f\left(e^{(n)}\right)\right)_{n=1}^{\infty} \text { and } b=f(e)-\sum_{n=1}^{\infty} a_{n} .
$$

Moreover, if $f \in X^{*}$, then

$$
\|f\|=|b|+\|a\|_{\mathscr{X}} .
$$

\section{Matrix transformations}

In this section, we characterise the classes $(X, Y)$ and $\left(X_{0}, Y\right)$ for $X \in\left\{c, w^{p},[c]\right\}$ and $Y \in\left\{c_{0}, c\right\}$.

Theorem 3.1 Let $X \in\left\{c, w^{p},[c]\right\}$. Then

(a) $A \in\left(X_{0}, c\right)$ if and only if

$$
\|A\|_{\left(X, \ell_{\infty}\right)}=\sup _{n}\left\|A_{n}\right\|_{\mathscr{X}}<\infty
$$

and

$$
\alpha_{k}=\lim _{n \rightarrow \infty} a_{n k} \text { exists for each } k
$$


$A \in\left(X_{0}, c_{0}\right)$ if and only if the condition in (4) holds and

$$
\lim _{n \rightarrow \infty} a_{n k}=0 \text { for each } k
$$

(b) $A \in(X, c)$ if and only if the conditions in (4) and (5) hold and

$$
\tilde{\alpha}=\lim _{n \rightarrow \infty} \sum_{k=1}^{\infty} a_{n k} \text { exists }
$$

$A \in\left(X, c_{0}\right)$ if and only if the conditions in (4) and (6) hold and

$$
\lim _{n \rightarrow \infty} \sum_{k=1}^{\infty} a_{n k}=0
$$

(c) If $A \in(X, Y)$ for $Y \in\left\{c_{0}, c\right\}$, then

$$
\left\|L_{A}\right\|=\|A\|_{\left(X, \ell_{\infty}\right)}
$$

(with $L_{A}$ from (1)).

Proof. Since $X_{0}$ and and $X$ are $B K$ spaces by Proposition 2.1 (a), it follows from [10, Theorem 9.3 .3 (c) (9.10)] that $A \in\left(Z, \ell_{\infty}\right)$ for $Z=X_{0}$ and $Z=X$ if and only if

$$
\|A\|_{\left(Z, \ell_{\infty}\right)}=\sup _{n}\left\|A_{n}\right\|_{Z}^{*}<\infty .
$$

We also have $\|\cdot\|_{Z}^{*}=\|\cdot\|_{\mathscr{X}}$ by Proposition 2.2 (a), so it follows from (10) that $A \in\left(Z, \ell_{\infty}\right)$ if and only if the condition in (4) is satisfied.

(a) Now let $Z=X_{0}$, a $B K$ space with $A K$ by Proposition 2.1 (b). Since $c$ and $c_{0}$ are closed subspaces of the $B K$ space $\ell_{\infty}$, we obtain from $[12,8.3 .6]$ that $A \in\left(X_{0}, Y\right)$ for $Y=c$ and $Y=c_{0}$ if and only if the condition in (4) holds in both cases, and $A^{k} \in Y$ for each $k$, which yields the conditions in (5) for $Y=c$ and in (6) for $Y=c_{0}$.

(b) Since $X=X_{0} \oplus e$, it follows from [12, 8.3.7] that $A \in(X, Y)$ for $Y=c$ or $Y=c_{0}$ if and only if $A \in\left(X_{0}, Y\right)$ and $A e \in Y$, which accounts for the additional conditions in accounts in (7)for $Y=c$ and (8).

(c) Part $c$ follows from [10, Theorem 9.3.3 (c) (9.11)].

Remark 3.2 (a) The case $X=c$ of Theorem 3.1 (b) is the famous classical Toeplitz theorem [11] (1911).

(b) Since $X_{0}$ has $A K$, Theorem 3.1 also characterises the classes of operators $L$ in $\mathscr{B}\left(X_{0}, c\right)$ and $\mathscr{B}\left(X_{0}, c_{0}\right)$ and provides a formula for the operator norm $\|L\|$, where $A$ is the matrix that represents $L$ as in (2). 


\section{The Hausdorff measure of noncompactness and compact operators}

In this section, we give estimates for the Hausdorff measure of noncompactness of the operators $L_{A}$ of the matrices $A \in(X, c)$, when $X \in\left\{c, w^{p},[c]\right\}$, characterise the classes $\mathscr{K}(X, c)$ of compact matrix operators in $(X, c)$, and apply our results to obtain that matrices in $(X, c)$ that preserve the limits cannot be compact matrix operators.

It is useful to recall the definitions of the Hausdorff measures of noncompactness of bounded sets in complete metric spaces and of operators between Banach spaces.

Definition 4.1 ([1, Definition 2.1]) Let $X$ be a complete metric space and $\mathscr{M}_{X}$ denote the class of all bounded subsets of $X$. Then the function $\chi: \mathscr{M}_{X} \rightarrow[0, \infty)$ with

$$
\chi(Q)=\inf \{\varepsilon>0: Q \text { has a finite } \varepsilon-\text { net in } X\}
$$

is called the Hausdorff or ball measure of noncompactness.

Definition 4.2 ([8, Definition 2.24]) Let $X$ and $Y$ be Banach spaces and $\chi$ be the Hausdorff measure of noncompactness.

(a) An operator $L: X \rightarrow Y$ is said to be $\chi$-bounded, if

$$
L(Q) \in \mathscr{M}_{Y} \text { for all } Q \in \mathscr{M}_{X},
$$

and if there exists a nonnegative real number $C$ such that

$$
\chi(L(Q)) \leq C \cdot \chi(Q) \text { for all } Q \in \mathscr{M}_{X} .
$$

(b) If an operator $L$ is $\chi$-bounded, then the number

$$
\|L\|_{\chi}=\inf \{C \geq 0:(11) \text { holds }\}
$$

is called the Hausdorff measure of noncompactness of $L$.

We need the following important, well-known results.

Theorem 4.3 (Goldenštein, Go'hberg, Markus) ([8, Theorem 2.23])

Let $X$ be a Banach space with a Schauder basis $\left(b_{n}\right)_{n=1}^{\infty}$ and the operator $\mathscr{R}_{n}: X \rightarrow X$ for each $n \in \mathbb{N}$ be defined by

$$
\mathscr{R}_{n}(x)=\sum_{k=n+1}^{\infty} \lambda_{k} b_{k} \text { for all } x=\sum_{k=1}^{\infty} \lambda_{k} b_{k} \in X .
$$

We put

$$
\mu(Q)=\limsup _{n \rightarrow \infty}\left(\sup _{x \in Q}\left\|\mathscr{R}_{n}(x)\right\|\right) \text { for all } Q \in \mathscr{M}_{X} .
$$


Then the following inequalities hold for all $Q \in \mathscr{M}_{X}$

$$
\frac{1}{a} \cdot \mu(Q) \leq \chi(Q) \leq \inf _{n}\left(\sup _{x \in Q}\left\|\mathscr{R}_{n}(x)\right\|\right) \leq \mu(Q),
$$

where $a=\limsup _{n \rightarrow \infty}\left\|\mathscr{R}_{n}\right\|$ is the basis constant of the Schauder basis.

Theorem 4.4 Let $X$ and $Y$ be $B K$ spaces and $L \in \mathscr{B}(X, Y)$.

Then we have

(a) ([8, Theorem 2.25])

$$
\|L\|_{\chi}=\chi\left(L\left(S_{X}\right)\right)=\chi\left(L\left(\bar{B}_{X}\right)\right)=\chi\left(L\left(B_{X}\right)\right),
$$

where $S_{X}, \bar{B}_{X}$ and $B_{X}$ denote the unit sphere, and the open and closed unit balls in $X$;

(b) $([8$, Corollary $2.26(2.58)])$

$$
\|L\|_{\chi}=0 \text { if and only if } L \in \mathscr{K}(X, Y) .
$$

Now we establish an estimate for the Hausdorff measure of noncompactness of operators $L_{A}$ for $A \in(X, c)$ when $X \in\left\{c, w^{p},[c]\right\}$.

Theorem 4.5 (a) Let $X \in\left\{c, w^{p},[c]\right\}$ and $A \in(X, c)$. Then

$$
\frac{1}{2} \limsup _{n \rightarrow \infty}\left(\left|\sum_{k=1}^{\infty} \alpha_{k}-\tilde{\alpha}\right|+\left\|\tilde{A}_{n}\right\|_{\mathscr{X}}\right) \leq\left\|L_{A}\right\|_{\chi} \leq \limsup _{n \rightarrow \infty}\left(\left|\sum_{k=1}^{\infty} \alpha_{k}-\tilde{\alpha}\right|+\left\|\tilde{A}_{n}\right\| \mathscr{X}\right),
$$

where $L_{A}$ is the operator as in (1), the numbers $\tilde{\alpha}$ and $\alpha_{k}(k \in \mathbb{N})$ are from (7) and (5), and $\tilde{A}=\left(\tilde{a}_{n k}\right)_{n, k=1}^{\infty}$ is the matrix with $\tilde{a}_{n k}=a_{n k}-\alpha_{k}$ for all $n$ and $k$.

(b) If $A \in(X, c)$, then $L_{A} \in \mathscr{K}(X, c)$ if and only if

$$
\lim _{n \rightarrow \infty}\left(\left|\sum_{k=1}^{\infty} \alpha_{k}-\tilde{\alpha}\right|+\left\|\tilde{A}_{n}\right\|_{\mathscr{X}}\right)=0
$$

Proof. We write $\|\cdot\|=\|\cdot\|_{X}$, for short.

Let $A \in(X, c)$. Then the numbers $\tilde{\alpha}$ and $\alpha_{k}(k \in \mathbb{N})$ exist by Theorem 3.1 (c). Also, since $X$ and $c$ are $B K$ spaces, $L_{A} \in \mathscr{B}(X, Y)$ by Theorem 1.1 (a).

(a) First we show

$$
\left(\alpha_{k}\right)_{k=1}^{\infty} \in \mathscr{X} .
$$

Since $X^{\beta}=X_{0}^{\beta}=\mathscr{X}$ by Proposition 2.2 (a), it suffices to show that $\left(\alpha_{k}\right)_{k=1}^{\infty} \in X_{0}^{\beta}$. It follows from the fact that $X_{0}$ has $A K$ by Proposition 2.1 (b) that there exists a positive constant $C$ such that $\left\|x^{[m]}\right\| \leq C\|x\|$, hence

$$
\left|\sum_{k=1}^{m} a_{n k} x_{k}\right|=\left|A_{n} x^{[m]}\right| \leq\left\|A_{n}\right\|_{X}^{*}\left\|x^{[m]}\right\| \leq C\|A\|_{\left(X, \ell_{\infty}\right)}\|x\| \text { for all } m \text { and } n,
$$


and so by (5) and (4)

$$
\left|\sum_{k=1}^{m} \alpha_{k} x_{k}\right|=\lim _{n \rightarrow \infty}\left|\sum_{k=1}^{m} a_{n k} x_{k}\right| \leq C\|A\|_{\left(X, \ell_{\infty}\right)}\|x\|<\infty \text { for all } m,
$$

that is, the sequence of partial sums $\sum_{k=1}^{m} \alpha_{k} x_{k}$ is bounded for each $x \in X_{0}$. Since $X_{0}$ has $A K$ this implies $\left(\alpha_{k}\right)_{k=1}^{\infty} \in X_{0}^{\beta}$ by [12, Theorem 7.2.7 (iii)].

Now we show

$$
\lim _{n \rightarrow \infty} A_{n} x=\sum_{k=1}^{\infty} \alpha_{k} x_{k} \text { for all } x \in X_{0} .
$$

Since $A \in\left(X_{0}, c\right)$ and $\left(\alpha_{k}\right)_{k=1}^{\infty} \in \mathscr{X}$ by (17), it follows for all $n$ that

$$
\left\|\tilde{A}_{n}\right\|_{\mathscr{X}} \leq\left\|A_{n}\right\|_{\mathscr{X}}+\left\|\left(\alpha_{k}\right)_{k=1}^{\infty}\right\|_{\mathscr{X}} \leq\|A\|_{\left(X, \ell_{\infty}\right)}+\left\|\left(\alpha_{k}\right)_{k=1}^{\infty}\right\|_{\mathscr{X}}
$$

hence the matrix $\tilde{A}$ satisfies the condition in (4). Also we have $\lim _{n \rightarrow \infty} \tilde{a}_{n k}=0$ for each $k$, that is, the matrix $\tilde{A}$ satisfies the condition in (6). Consequently $\tilde{A} \in\left(X_{0}, c_{0}\right)$ by Theorem 3.1 (a). Since $\left(\alpha_{k}\right)_{k=1}^{\infty} \in X_{0}^{\beta}$, we obtain (18).

Now let $x \in X$ and $y=A x$. Then $x^{(0)}-\xi_{X} e \in X_{0}$. Since $A_{n} \in X^{\beta}$ for all $n$, and $e \in X$, we have

$$
y_{n}=A_{n} x=A_{n}\left(x^{(0)}-\xi_{X} e\right)=A_{n} x^{(0)}+\xi A_{n} e \text { for all } n,
$$

and it follows from (18), (7) and (5) that

$$
\eta=\lim _{n \rightarrow \infty} A_{n} x=\sum_{k=1}^{\infty} \alpha_{k} x_{k}^{(0)}+\xi_{k} \lim _{n \rightarrow \infty} \sum_{k=1}^{\infty} a_{n k}=\sum_{k=1}^{\infty} \alpha_{k} x_{k}+\xi_{X}\left(\tilde{\alpha}-\sum_{k=1}^{\infty} \alpha_{k}\right) .
$$

Now we have for all $m$

$$
\mathscr{R}_{m}(y)=\sum_{n=m+1}^{\infty}\left(y_{n}-\eta\right) e^{(n)}
$$

We write $f_{n}^{(m)}(x)=\left(\left(\mathscr{R}_{m} \circ L_{A}\right)(x)\right)_{n}$ for all $n$ and $m$, and obtain for $n \geq m+1$ by (19)

$$
f_{n}^{(m)}(x)=A_{n} x-\sum_{k=1}^{\infty} \alpha_{k} x_{k}+\xi_{X}\left(\sum_{k=1}^{\infty} \alpha_{k}-\tilde{\alpha}\right)=\tilde{A}_{n} x+\xi_{X}\left(\sum_{k=1}^{\infty} \alpha_{k}-\tilde{\alpha}\right) .
$$

Since $f_{n}^{(m)} \in c^{*}$, it follows by (3) that

$$
\sup _{x \in S_{X}}\left\|\mathscr{R}_{m}\left(L_{A}(x)\right)\right\|_{\infty}=\sup _{n \geq m+1}\left\|f_{n}^{(m)}\right\|=\sup _{n \geq m+1}\left(\left|\sum_{k=1}^{\infty} \alpha_{k}-\tilde{\alpha}\right|+\left\|\tilde{A}_{n}\right\| \mathscr{X}\right) .
$$

Finally it follows from (12), (13) and the fact that $a=\limsup _{m \rightarrow \infty}\left\|\mathscr{R}_{m}\right\|=2$ by [10, Example 7.9.7]

$$
\frac{1}{2} \inf _{m}\left\|f_{n}^{(m)}\right\|=\frac{1}{2} \inf _{m}\left(\sup _{n \geq m+1}\left|\sum_{k=1}^{\infty} \alpha_{k}-\tilde{\alpha}\right|+\left\|\tilde{A}_{n}\right\|_{\mathscr{X}}\right)
$$




$$
=\frac{1}{2} \limsup _{m \rightarrow \infty}\left(\left|\sum_{k=1}^{\infty} \alpha_{k}-\tilde{\alpha}\right|+\left\|\tilde{A}_{m}\right\|_{\mathscr{X}}\right) \leq\left\|L_{A}\right\|_{\chi} \leq \inf _{m}\left\|f_{n}^{(m)}\right\| .
$$

This yields the inequalities in (15).

(b) Part (b) is an immediate consequence of (15) and (14).

Now we generalise the concept of regularity to that of $X$-regularity and apply Theorem 4.5 to conclude that an $X$-regular matrix transformation cannot be compact.

Again let $X \in\left\{c, w^{p},[c]\right\}$. A matrix $A \in(X, c)$ is said to be $X$-regular, if $\lim _{n \rightarrow \infty} A_{n} x=$ $\xi_{X}$ for all $x \in X$, where $\xi_{X}$ is the $X$-limit of the sequence $x$. If $X=c$, then $X$-regularity is the usual regularity.

Corollary 4.6 An X-regular matrix operator cannot be compact.

Proof. Let $A \in(X, c)$ be $X$-regular. Then it follows from (19) that

$$
\lim _{n \rightarrow \infty} A_{n} e^{(k)}=\lim _{n \rightarrow \infty} a_{n k}=\alpha_{k}=0 \text { for each } k, \text { and } \lim _{n \rightarrow \infty} A_{n} e=\tilde{\alpha}=1,
$$

hence $1+\left\|A_{n}\right\|_{\mathscr{X}} \geq 1$ for all $n$, and so (16) is not satisfied.

Remark 4.7 For $X=c$, Corollary 4.6 reduces to the known result of Cohen and Dunford [2] that a regular matrix transformation cannot be compact.

Acknowledgement. Dedicated to Professor Dolićanin on the occasion of his 75th birthday.

\section{References}

[1] J. M. Ayerbe Toledano, T. Dominguez Benavides and G. Lopez Acedo, Measures of Noncompactness in Metric Fixed Point Theory, Operator Theory Advances and Applications , Vol. 99 Birkhäuser Verlag, Basel, Boston, Berlin 1997

[2] L. W. Cohen and N. Dunford, Transformations on sequence spaces, Duke Math. J., Vol 3, no. 4 (1937), 689-701

[3] A. M. JARRAH and E. MALKOWSKy, Ordinary, absolute and strong summability and matrix transformations, Filomat, Vol. 17 (2003), 59-78

[4] B. Kuttner and B. Thorpe, Strong convergence, J. Reine Angew. Math., Vol 311/312 (1979), 42-55

[5] I. J. Maddox, On Kuttner's theorem, London J. Math. Soc., Vol. 43 (1968), 285-298

[6] E. MaLKowsky, The continuous duals of the spaces $c_{0}(\Lambda)$ and $c(\Lambda)$ for exponentially bounded sequences $\Lambda$, Acta Sci. Math (Szeged), Vol. 61 (1995), 241-250

[7] E. Malkowsky, The dual space of the sets of $\Lambda$-strongly convergent and bounded sequences, Novi Sad J. Math., Vol 30 no. 3 (2000), 99-110

[8] E. Malkowsky and V. RakočEVić, An introduction into the theory of sequence spaces and measures of noncompactness, In: Zbornik radova, Matematčki institut SANU, Vol. 9(17), Mathematical Institute of SANU, Belgrade 2000, pp. 143-234 
[9] E. MALKOWSKY and V. RAKOČEVIĆ, On some results using measures of noncompactness, In: Advances in Nonlinear Analysis via the Concept of Measure of Noncompactness ( $\mathrm{J}$. Banaś, M. Mursaleen et al. eds.) Springer Verlag, 2017, pp. 127-180

[10] E. Malkowsky and V. Rakočević, Advanced Functional Analysis, CRC Press, Taylor and Francis Group, Chapman \& Hall, 6000 Broken Sound Parkway NW, Suite 300, Boca Raton, FL 33487, USA, 2019

[11] O. Toeplitz, Über allgemeine Mittelbildungen, Prace. Mat. Fiz., Vol. 22 (1911) 113-119

[12] A. Wilansky, Summability through Functional Analysis, North-Holland, Mathematical Studies, Amsterdam, 1984 